\title{
Optimal energy management for industrial microgrids with high-penetration renewables
}

\author{
Han Li ${ }^{1}$, Abinet Tesfaye Eseye ${ }^{2,3^{*}} \mathbb{D}$, Jianhua Zhang ${ }^{2}$ and Dehua Zheng ${ }^{3}$
}

\begin{abstract}
This paper presents a day-ahead optimal energy management strategy for economic operation of industrial microgrids with high-penetration renewables under both isolated and grid-connected operation modes. The approach is based on a regrouping particle swarm optimization (RegPSO) formulated over a day-ahead scheduling horizon with one hour time step, taking into account forecasted renewable energy generations and electrical load demands. Besides satisfying its local energy demands, the microgrid considered in this paper (a real industrial microgrid, "Goldwind Smart Microgrid System" in Beijing, China), participates in energy trading with the main grid; it can either sell power to the main grid or buy from the main grid. Performance objectives include minimization of fuel cost, operation and maintenance costs and energy purchasing expenses from the main grid, and maximization of financial profit from energy selling revenues to the main grid. Simulation results demonstrate the effectiveness of various aspects of the proposed strategy in different scenarios. To validate the performance of the proposed strategy, obtained results are compared to a genetic algorithm (GA) based reference energy management approach and confirmed that the RegPSO based strategy was able to find a global optimal solution in considerably less computation time than the GA based reference approach.
\end{abstract}

Keywords: Energy management, Genetic algorithm, Microgrid, Regrouping particle swarm optimization, Renewable energy

\section{Introduction}

Microgrids are a group of interconnected loads, distributed energy resources (including conventional energy sources and renewables) and energy storage systems at a distribution level with distinct electrical boundaries. A microgrid has black start capability and can operate either in isolated or non-isolated mode in connection with other microgrids or main electricity grid.

Non-isolated (grid-connected) microgrids can either send (sell) power to the main grid or receive (buy) from the main grid. This electric power trading with the main grid has traditionally been based on a fixed, pre-determined price per $\mathrm{kWh}$. However, with the incorporation of smart meter technologies, capable of accurately measuring energy production and consumption in each time instant, a shift to

\footnotetext{
* Correspondence: abinet47@yahoo.com

${ }^{2}$ School of Electrical and Electronic Engineering, North China Electric Power

University, Changping District, Beijing 102206, CN, China

${ }^{3}$ Goldwind Science and Etechwin Electric Co., Ltd., Beijing

Economic-Technology Development Area, Beijing 100176, CN, China

Full list of author information is available at the end of the article
}

time-varying electricity pricing models is being occurred recently [1]. Advanced control technologies that can combine together several generation systems and energy storage systems in microgrid entity are emerging to offer customers the opportunity to access reliable and secured electricity locally, sell power during surplus generation or peak grid price time periods, and buy power in case of generation scarcity or cheap electricity prices time instants.

This energy exchange strategy development motivates microgrid operators to adapt their energy trading actions with the main grid and/or other microgrids according to the current electricity price and trading conditions in order to minimize energy production running cost (fuel cost), ensure maximum utilization of renewables, maximize economic benefits of the energy storage systems. To achieve this, specific energy management system should have to be put in place [1-4].

The topic of optimization (cost minimization or profit maximization) in microgrids through energy management 
has already been dealt with by several researchers in different contexts.

An energy management model, with sensitivity analysis for energy storage capacity investment and electricity load demand growth, for searching optimum operating policies for maximization of profit in a microgrid system in Taiwan is presented in [5]. In [6], the minimization of total costs for energy production and transportation of two interconnected microgrids that can trade electric energy with each other but not connected to the main power grid is addressed. For this aim, a distributed and a central control strategy are examined using an iterative approach and an analytical convex optimization method.

Concerning the issue of energy exchange of a microgrid with the main power grid, [7] targets on the development of a neural network based energy management system (EMS) to allocate the dispatch of generation sources in a microgrid to take part in the energy trading market and minimize global energy production costs. Reference [8] introduces an energy control apparatus called "Energy Box" for controlling residential home or small business electrical energy utilization in an environment of demand sensitive real-time electricity pricing. A stochastic dynamic programming method is employed based on forecast information from load demands, weather, and grid price for optimally managing the utilization, storage and selling/buying of electrical energy. Reference [9] suggests an optimization model based on hierarchical control for a microgrid configuration capable of participating to the wholesale energy trading market as both energy consumer and producer with the objective of minimizing energy production costs and maximizing energy trading revenues.
Reference [10] proposes a generic mixed integer linear programming technique for operating cost minimization in market-based price environments for a residential microgrid including electrical and thermal loads, energy storage units and some controllable loads. Reference [11] presents an online optimal energy/power control strategy for the operation of energy storage in grid-connected microgrids. The approach is based on a mixed-integer-linear-programming formulated over a rolling horizon window, considering predicted future electricity load demands and renewable energy generations.

Reference [12] presents a genetic algorithm (GA) for optimal unit sizing of an isolated microgrid considering multiple objectives including life-cycle cost minimization, renewable energy penetration maximization, and emission reduction. In [13, 14], particle swarm optimization (PSO) has been applied for real-time energy management of stand-alone microgrids.

In most of the literatures reported above, regarding energy management strategies in microgrids, a single energy storage unit is considered. The integration and combined optimal storage management of microgrids containing two or more energy storage units (ESUs) have not been considered so far. Moreover, the PSO is seen to suffer from stagnation once particles have prematurely converged to any particular region of the search space in the energy management strategies that have applied the standard version of PSO for solving the energy management optimization problem [15].

An actual industrial microgrid (Goldwind Smart Microgrid System), in Beijing, China, is considered to deliver the power demand requirements of the various loads within an industrial park (Goldwind Science and Etechwin Electric. Co., Ltd.), shown in Fig. 1. It comprises of wind

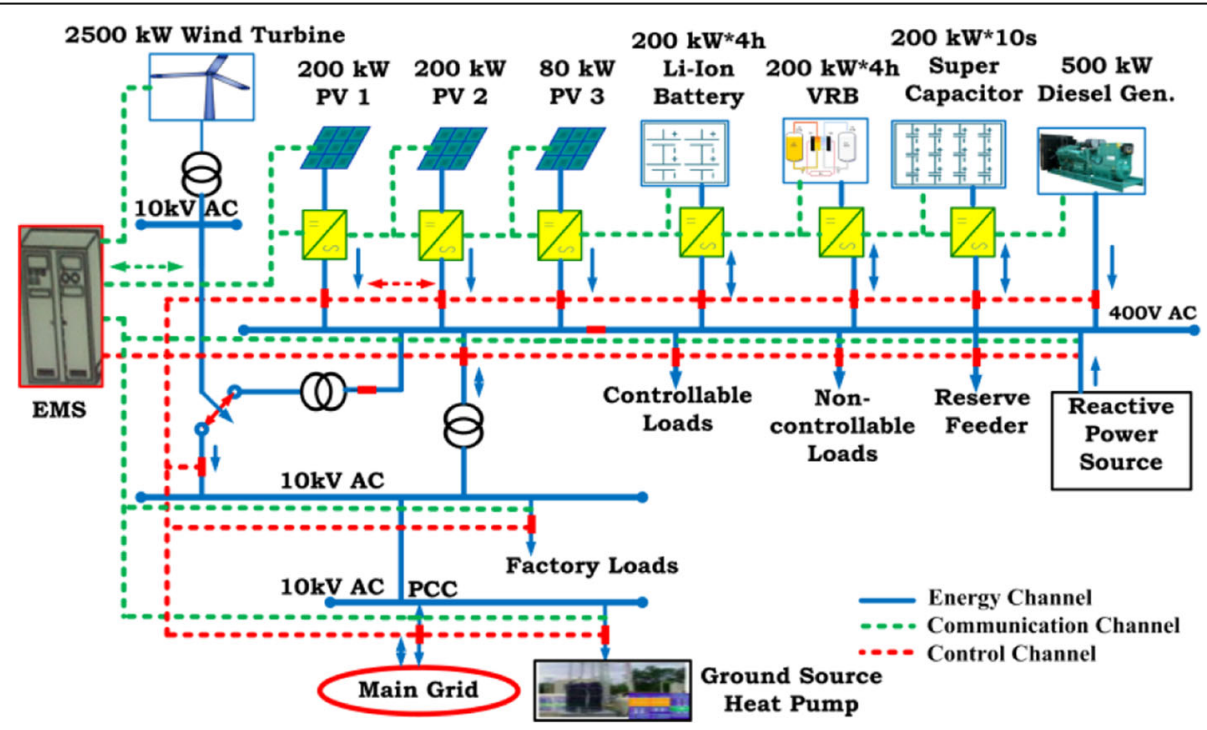

Fig. 1 Microgrid architecture and system model 
energy conversion system (WECS) that utilizes a permanent magnet synchronous generator (PMSG), three solar PV systems, diesel generator (DE) and energy storage system (ESS) containing two storage batteries, vanadium redox flow battery (VRB) and lithium-ion (Li-Ion) battery. The super capacitor energy storage, shown in Fig. 1, is used for transient energy balance compensation, not for steady state energy storage, and hence it is not included in the optimization model. The microgrid is connected to the main grid through a $10 \mathrm{kV}$ bus at the point of common coupling (PCC). The microgrid operates under both isolated and non-isolated modes. The microgrid is in islanded mode when the power switch (PCC main switch) between the PCC bus and the main grid is disconnected and in non-isolated mode when this switch is turned on. The actual ratings of the components as shown in Fig. 1 are used in this paper.

In this paper, we propose a RegPSO approach to optimally solve the EMS optimization model. To evaluate and compare the performances of this approach, another modern optimization method, genetic algorithm (GA) was also implemented.

The rest of the paper is organized as follows. Section II discusses the formulations of the objective and constraint functions. In Section III, the proposed method of optimal energy management strategy and the RegPSO algorithm are presented. The case study simulation results are discussed and performance comparisons are provided in Section IV, and finally the paper is concluded in Section V.

\section{Discussion}

\subsection{Microgrid energy management optimization model}

The objective problem and constraint functions of the optimization model for energy management in the microgrid considering the two possible operation modes are formulated in this section. In the isolated mode, the microgrid objective is formulated to minimize the energy production cost (fuel cost), and the operation and maintenance costs within the microgrid. While operating in grid-connected mode, the microgrid can either send (sell) power to the main grid or receive (buy) from the main grid. During the periods receiving power from the main grid, the microgrid is expected to minimize the energy production cost, operation and maintenance cost and the expense of buying power from the main grid; while sending power to the main grid, the objective is to maximize the profit which is the energy selling revenue minus the energy production cost, and operation and maintenance cost.

This objective function is subjected to six decision variables: the charging/discharging power of the VRB, state of charge (SOC) of the VRB, charging/ discharging power of the Li-Ion battery, SOC of the Li-Ion battery, the diesel generator power output, and the quantity of power exchange with the main grid.

\subsection{Formulation of objective functions}

The following are some of the information that should be specified in advance for a day-ahead energy management in microgrids $[16,17]$ :

- 24-h-ahead hourly load demand forecast

- 24-h-ahead hourly wind power forecast

- 24-h-ahead hourly PV power forecast

- Grid price forecast, or pre-specified grid price

The objective functions are formulated independently by considering three operational cases based on the microgrid operating mode and the power flow directions between the microgrid and the main grid. In case I, the objective function for the isolated mode of operation is considered. In case II, the microgrid is in grid-connected mode and it receives (buys) power from the main grid. While in case III, the microgrid is also in grid-connected mode but it sends (sells) power to the main grid.

\subsubsection{Case I - isolated mode}

In case I, the objective targets to minimize the energy production cost (fuel cost), and the operation and maintenance costs within the microgrid.

The objective function is given by:

$$
\operatorname{Min} \sum_{t=1}^{n}\left\{\begin{array}{c}
\sum_{i=1}^{m}\left(F_{i}\left(P_{i}(t)\right) \cdot \tau_{i}(t)+S C_{i}(t)\right)+ \\
\sum_{i=1}^{m} C_{O M, i}(t) P_{i}(t)+C_{\text {OMwind }}(t) P_{\text {wind }}(t)+ \\
C_{\text {OMpv }}(t) P p v(t)+\sum_{j=1}^{q} C_{\text {OMes.j }}(t) P_{\text {es }, j}(t)
\end{array}\right\}
$$

Where, $\mathrm{n}$ is the number of time steps for a scheduling day; $m$ indicates the number of all types of dispatchable DGs; q is the number of all types of energy storage units within the microgrid; $P_{i}(t)$ is the power output of the $\mathrm{i}^{\text {th }}$ dispatchable DG at time $\mathrm{t}$ and $F_{i}\left(P_{i}(t)\right)$ is the corresponding fuel cost function, and for a diesel generator it is defined as:

$$
F_{i}\left(P_{i}(t)\right)=a_{i} \cdot P_{i}(t)^{2}+b_{i} \cdot P_{i}(t)+c
$$

Where, $a_{i}, b_{i}$ and $c_{i}$ are the cost function parameters.

$\tau_{i}(t)=1$, if the $\mathrm{i}^{\text {th }}$ dispatchable DG is in operation;

$\tau_{i}(t)=0$, if the $\mathrm{i}^{\text {th }}$ dispatchable DG is OFF at time $\mathrm{t}$;

$S C_{i}(t)$ is the start up cost function of each dispatchable DG and is given by: 


$$
\begin{aligned}
& S C_{i}(t)=s c_{i}, \text { if } \tau_{i}(t)-\tau_{i}(t-1)=1 \\
& S C_{i}(t)=0,
\end{aligned}
$$

otherwise

Where, $s c_{i}$ is the start up cost of dispatchable DG i.

$c_{O M, i}(t)$ is the operation and maintenance cost of the $\mathrm{i}^{\text {th }}$ dispatchable DG at time $\mathrm{t}$; $c_{\text {OMwind }}(t)$ is the operation and maintenance cost of the wind power generation system at time $\mathrm{t} ; P_{\text {wind }}(t)$ is the forecasted wind generation at time t; $c_{O M p v}(t)$ is the operation and maintenance cost of the PV system at time t; $P_{p \nu}(t)$ is the forecasted PV generation at time $\mathrm{t} ; C_{\text {OMes }, j}(t)$ is the operation and maintenance cost of the $j^{\text {th }}$ energy storage unit at time t; $P_{e s, j}(t)$ is the $\mathrm{j}^{\text {th }}$ energy storage charging/discharging power at time $t$.

\subsubsection{Case II - Non-isolated mode - buying power from main grid}

In this case, the objective aims in minimizing the energy production cost, the operation and maintenance costs and the expenses of energy purchasing from the main grid.

The objective function is:

$$
\operatorname{Min} \sum_{t=1}^{n}\left\{\begin{array}{c}
c_{\text {gridbuy }}(t) P_{\text {grid }}(t)+\sum_{i=1}^{m}\left(F_{i}\left(P_{i}(t)\right) \cdot \tau_{i}(t)+S C_{i}(t)\right)+ \\
\sum_{i=1}^{m} C_{O M, i}(t) P_{i}(t)+C_{\text {OMwind }}(t) P_{\text {wind }}(t)+ \\
C_{\text {OMpv }}(t) \operatorname{Ppv}(t)+\sum_{j=1}^{q} C_{\text {OMes. } j}(t) P_{\text {es } j,}(t)
\end{array}\right\}
$$

Where, $c_{\text {gridbuy }}(t)$ is the electricity buying price from the main grid at time $t ; P_{\text {grid }}(t)$ is the power purchased from the main grid at time $t, P_{\text {grid }}(t)>0$.

2.2.3 Case III - Non-isolated mode - selling power to main grid Here, the objective aims in maximizing the profit which is the energy selling revenue minus the energy production cost and the operation and maintenance costs within the microgrid.

The objective function becomes

$$
\left.\operatorname{Max}_{t=1}^{n}\left\{\begin{array}{c}
-\sum_{\text {gridsell }}(t) P_{\text {grid }}(t)- \\
\sum_{i=1}^{m}\left(F_{i}\left(P_{i}(t)\right) \cdot \tau_{i}(t)+S C_{i}(t)\right)+\sum_{i=1}^{m} C_{\text {OM }, i}(t) P_{i}(t)+ \\
C_{\text {OMwind }}(t) P_{\text {wind }}(t)+C_{\text {OMpv }}(t) P p v(t)+ \\
\sum_{j=1}^{q} C_{\text {OMes } j}(t) P_{\text {ess }}(t)
\end{array}\right\}\right)
$$

Where, $c_{\text {gridsell }}(t)$ is the electricity selling price to the main grid at time t; $P_{\text {grid }}(t)$ is the power sold to the main grid at time $\mathrm{t}, P_{\text {grid }}(t)<0$.

\subsection{Formulation of constraint functions}

The objective functions formulated above are subjected to the following constraints; comprising ESS units' capacity and operational limits, dispatchable DGs' power limit, grid power transfer limits, and all other technical requirements in the microgrid:

\subsubsection{Power output of the $i^{\text {th }}$ dispatchable DG at time $t$}

$$
P_{i}^{\min }(t) \leq P_{i}(t) \leq P_{i}^{\max }(t)
$$

\subsubsection{Grid power exchange limits}

$$
P_{\text {grid }}^{\min }(t) \leq P_{\text {grid }}(t) \leq P_{\text {grid }}^{\max }(t)
$$

The grid power exchange minimum $\left(P_{\text {grid }}^{\min }(t)\right)$ and maximum $\left(P_{\text {grid }}^{\max }(t)\right)$ limits can be set as a large amount or the capacity of the transformer linking the microgrid and the main grid.

\subsubsection{Demand-supply balance}

$$
\sum_{i=1}^{m} P_{i}(t)+\sum_{i=j}^{q} P_{e s, j}(t)=P_{\text {load }}(t)-P_{\text {wind }}(t)-P_{p v}(t)-P_{\text {grid }}(t)
$$

where $P_{\text {load }}(t)$ denotes the forecasted load demands at time t.

\subsubsection{ESS units charging/discharging power limits}

$$
P_{e s, j}^{\min }(t) \leq P_{e s, j}(t) \leq P_{e s, j}^{\max }(t)
$$

$P_{e s, j}(t)>0$, the $\mathrm{i}^{\text {th }}$ energy storage is discharging;

$P_{e s, j}(t)<0$, the $\mathrm{i}^{\text {th }}$ energy storage is charging;

$P_{e s, j}(t)=0$, the $\mathrm{i}^{\text {th }}$ energy storage is inactive.

\subsubsection{ESS units dynamic operation performance}

$$
\begin{aligned}
& S O C_{e s, j}(t+1)=S O C_{e s, j}(t)-\frac{\eta_{e s, j}(t) P_{e s, j}(t)}{C_{e s, j}} \\
& S O C_{e s, j}^{\min } \leq S O C_{e s, j}(t+1) \leq S O C_{e s, j}^{\max }
\end{aligned}
$$

Where, $\eta_{e s, j}(t)$ is the $\mathrm{i}^{\text {th }}$ energy storage unit charging or discharging efficiency at time $t$; $C_{e s, j}$ denotes the rated storage capacity of $j^{\text {th }}$ energy storage unit.

Thus, the decision variables that need to be determined are the ESUs' charging/discharging power $P_{e s, j}(t)$ and their state of charges $S O C_{e s, j}(t)$ (for $\mathrm{i}=1$, $2, \ldots, \mathrm{q})$; the power output of dispatchable DGs $P_{i}(t)$,and the quantity of power exchange with the main grid $P_{\text {grid }}(t)$ at time t. 


\section{Method}

\subsection{Proposed microgrid energy management strategy}

The purpose of the EMS is to make secure day-ahead decisions for the microgrid economic operations. The proposed system takes into account the intermittency of renewable generations, the fluctuations of load demands, the energy production fuel cost, operation and maintenance costs of different sources, technical restrictions and capacity limits, time-varying grid prices, and the energy trading revenue possibilities with the main grid. Figure 2 illustrates the information flows of the proposed system, in which an EMS converts to output commands for energy storage units' optimal charging/discharging power, diesel generator power output, and main grid import/export power quantities. Decisions are based primarily on renewable generation and load demand forecasts, energy production fuel cost and electricity prices in the main grid. These decisions are generated in this paper for a day-ahead time horizon on 1 hour interval basis.

The EMS in this study is restricted to control only the real power. Power quality, frequency regulation, and voltage stability are supposed to be controlled at the

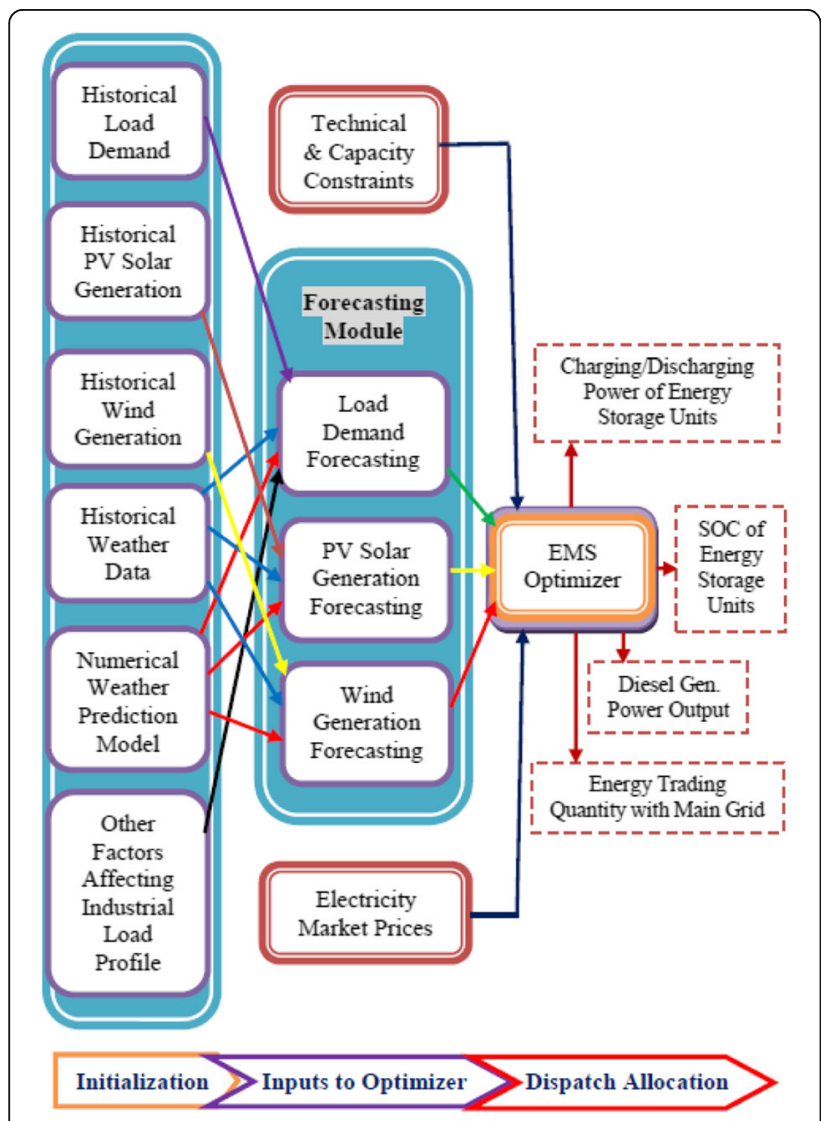

Fig. 2 Information flow in the proposed EMS generation level. Microgrid black start operation or synchronization with the main grid is not considered either. The proposed system comprises functions, such as an energy storage units charging/discharging power economic scheduling, diesel generator output power optimal scheduling, forecasting for renewable generators and load demands, and energy trading participation with the main grid.

\subsection{The RegPSO algorithm}

PSO has few variables to update and is simple to implement. Many researches and applications have been successfully implemented using the PSO concept. Reference [18] presents a general idea of PSO and its applications in power systems, and also gives comparisons with other optimization methods.

For a decision vector $x \in \Re^{n}$ consisting the objective problem's decision variables (positions), the feasible design search space is defined by a subset [17]:

$$
\Omega=\left[x_{1}^{L}, x_{1}^{U}\right] \times\left[x_{2}^{L}, x_{2}^{U}\right] \times \ldots \times\left[x_{n}^{L}, x_{n}^{U}\right] \subset \boldsymbol{R}^{n}
$$

where $x_{j}^{L}$ and $x_{j}^{U}$ are, respectively, the lower and upper bounds of the design search space along dimension $j$ for $\mathrm{j}=1,2, \ldots, \mathrm{n}$.

The position or coordinate of the $\mathrm{i}^{\text {th }}$ particle in the $\mathrm{k}^{\text {th }}$ iteration is give by:

$$
x_{i}(k)=x_{i}(k-1)+v_{i}(k) ; i=1,2, \ldots, N
$$

where $\mathrm{N}$ is the swarm size and $v_{i}(k)$ is the velocity of the $\mathrm{i}^{\text {th }}$ particle at the $\mathrm{k}^{\text {th }}$ iteration which shows the rate of change of particle's position in the design search space, and given by:

$$
\begin{aligned}
& v_{i}(k)=\omega(k) v_{i}(k-1)+c_{1} r_{1}\left(P_{\text {best }, i}-x_{i}(k-1)\right) \\
& +c_{2} r_{2}\left(G_{\text {best }}-x_{i}(k-1)\right)
\end{aligned}
$$

where, $\omega(\mathrm{k})$ is a dynamic inertia weight whose value declines linearly with the iteration number to dampen the velocities over iterations, enabling the swarm to converge more precisely and efficiently, and given by:

$$
\omega(k)=\omega_{\max }-\left(\frac{\omega_{\max }-\omega_{\min }}{k_{\max }}\right) \cdot k
$$

where $\omega_{\max }$ and $\omega_{\min }$ are the initial and final inertia weight values, respectively, $k_{\max }$ is the maximum number of iterations used; $c_{1}$ and $c_{2}$ are the cognitive and social learning rates respectively, and $r_{1}$ and $r_{2}$ are random numbers in the range of 0 and 1 . The parameters $c_{1}$ and $c_{2}$ represent the relative importance of the position (memory) of the particle itself to the position (memory) of the swarm; pBest or $P_{b e s t, i}$ is the best position achieved so for by particle i, while 
gBest or $G_{\text {best }}$ is the global best position of all the particles in the swarm.

However, PSO is seen to suffer from stagnation when particles have prematurely converged to any specific region of the design search space [15]. The proposed RegPSO circumvents this stagnation problem by automatically reorganizing the swarm when premature convergence is detected or when the maximum number of iterations or function evaluations per grouping is reached [19]; this liberates particles in the swarm from the state of premature convergence, thus enabling continued exploration toward the true global minimum solution. This is computationally simple yet effective improvement to the conventional PSO algorithm. RegPSO has been tested experimentally over popular benchmark optimization problems and successfully approximates the global minimum of these benchmark problems [15]. The flowchart in Fig. 3 shows the general working principle of the RegPSO algorithm.

At each iteration, $\mathrm{k}$, the swarm radius, $\delta(\mathrm{k})$, is considered to be the maximum Euclidean distance, in $n$-dimensional search space, of any particle from the global best solution as follows:

$$
\delta(k)=\max _{i \in\{1,2, \ldots, N\}}\left\|x_{i}(k)-G_{b e s t}\right\|
$$

where $\|$.$\| represents the Euclidean norm.$

Let $\operatorname{diam}(\Omega)=\|\operatorname{range}(\Omega)\|$ be the diameter of the design search space. Particles are considered to be in proximity and regrouping is activated when the normalized swarm radius, $\delta_{\text {norm }}$, satisfies the premature convergence condition defined as:

$$
\delta_{\text {norm }}=\frac{\delta(k)}{\operatorname{diam}(\Omega)}<\varepsilon
$$

where $\varepsilon$, called the stagnation threshold.

When premature convergence is noticed as given by condition (15), the swarm is regrouped in a designed search space centered about the global best solution. The regrouping factor found to work well across benchmarks tested [15], given by (16), is constant across groupings.

$$
\rho<\frac{6}{5 \varepsilon}
$$

Upon detection of premature convergence, the range in which particles are to be regrouped about the global best is computed per dimension as the minimum of (i) the original range of the design search space on dimension $j$ and (ii) the product of the regrouping factor with the maximum distance along dimension $j$ of any particle from global best:

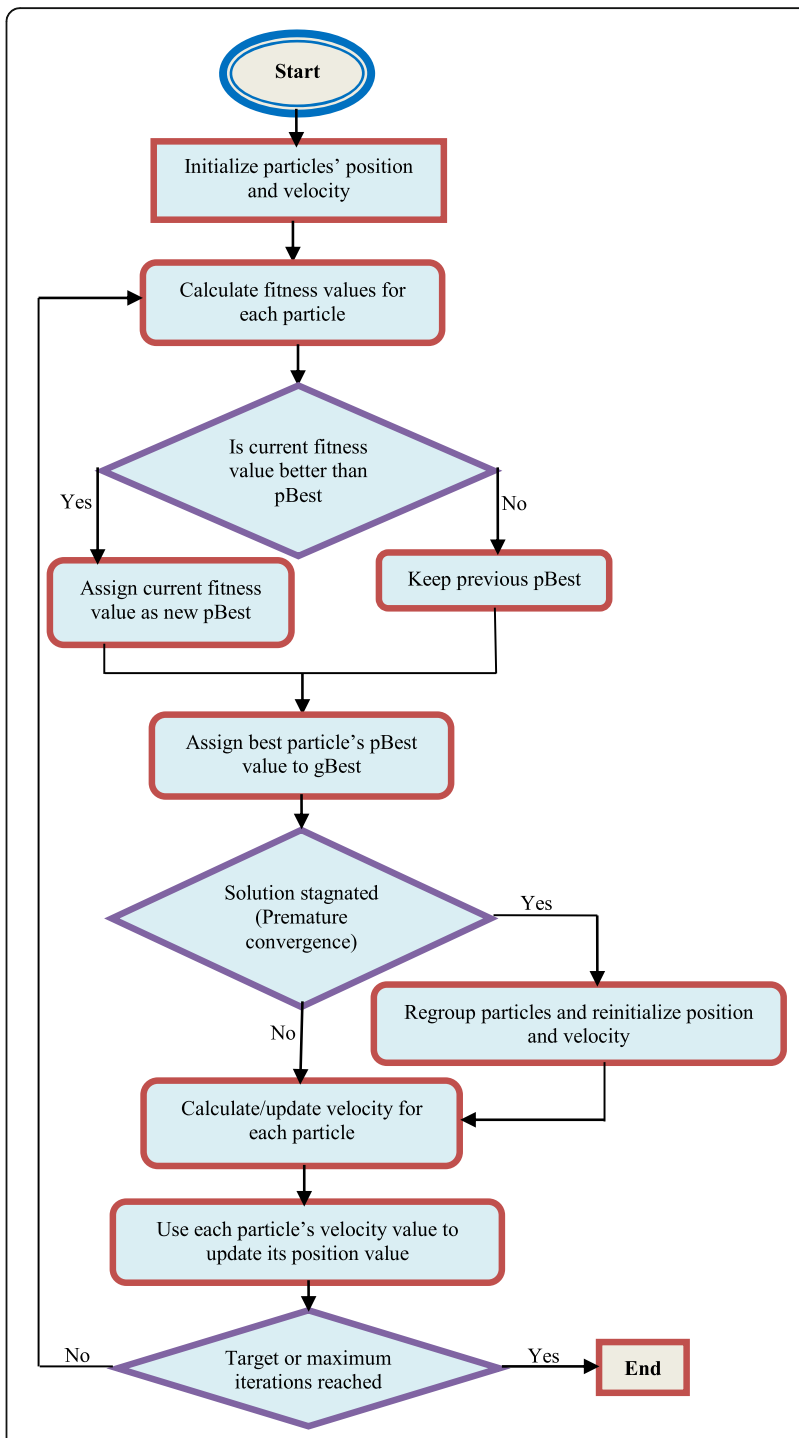

Fig. 3 Flowchart of the RegPSO algorithm

$$
\operatorname{range}_{j}\left(\Omega^{r}\right)^{j}=\min \left(\operatorname{range}_{j}\left(\Omega^{0}\right)^{j}, \rho \underset{i \in\{1, \ldots, N\}}{\max }\left|x_{i, j}^{r-1}-G_{\text {best }, j}^{r-1}\right|\right)
$$

The swarm is then regrouped by reinitializing particles' position as:

$$
x_{i}=G_{\text {best }}{ }^{-1}+\vec{r}^{\prime} \cdot \operatorname{range}\left(\Omega^{r}\right)-\frac{1}{2} \operatorname{range}\left(\Omega^{r}\right)
$$

where, $\operatorname{range}\left(\Omega^{r}\right)=\left[\operatorname{range}_{1}\left(\Omega^{r}\right), \ldots, \operatorname{range}_{n}\left(\Omega^{r}\right)\right]$ which utilizes a random vector $\vec{r}^{\prime}$ to randomize particles within the implicitly defined design search space:

$$
\Omega^{r}=\left[x_{1^{L, r}}, x_{1}^{u, r}\right] \times\left[x_{2^{L, r}}, x_{2^{u, r}}\right] \times \ldots \times\left[x_{n^{L, r}}, x_{n^{u, r}}\right]
$$

with respective lower and upper bounds as: 


$$
\begin{aligned}
x_{j} L, r & =G_{\text {best }, j}^{r-1}-\frac{1}{2} \operatorname{range}_{j}\left(\Omega^{r}\right)^{j} \\
x_{j} L, U & =G_{\text {best }, j}^{r-1}+\frac{1}{2} \operatorname{range}_{j}\left(\Omega^{r}\right)^{j}
\end{aligned}
$$

The swarm regrouping index, $r$, begins with 0 prior to the incidence of any regrouping and grows by one with each successive regrouping. Vector $G_{b e s t^{r-1}}$ is the global best at the last iteration of the previous grouping, and $\vec{x}_{i^{r-1}}$ is the position of particle $\mathrm{i}$ at the last iteration of the previous grouping. Note that before any regrouping takes place, the original design search space, $\Omega^{0}$, correspond to a swarm regrouping index of $r=0$. The maximum velocity is recomputed with each regrouping:

$$
v_{j} \max , r=\lambda \cdot \operatorname{range}_{j}\left(\Omega^{r}\right)^{j}
$$

Where, $\lambda$ is the velocity clamping factor.

\section{Result}

\subsection{Test case}

The $2500 \mathrm{~kW}$ wind, $480 \mathrm{~kW} \mathrm{PV,} 500 \mathrm{~kW}$ diesel generator, $4 \mathrm{~h} * 300 \mathrm{~kW}$ VRB, $4 \mathrm{~h} 200 \mathrm{~kW}$ Li-Ion battery industrial microgrid in this study is designed to deliver power to an industrial company. The minimum and maximum SOC of the ESUs is 20 and 100\%, respectively. An ideal 100\% charging/discharging efficiency is considered for all ESUs. The diesel generator fuel cost function parameters are 0.00025 $(\$ / k W h)^{2}, 0.0156 \$ / k W h, 0.3312 \$ / h$, and $23 \$$, for a, b, c, and $\mathrm{SC}$, respectively. The day-ahead forecasts for the wind and PV generation are shown in Figs. 4 and 5, respectively.

In China, Beijing, there is a three-step time-dependent tariff for buying electricity from the main grid (State grid) within a day. In 2016, this energy buying price for industrial companies in business development area (BDA-Yizhuang in Beijing) is: 5.7323 USD cents per kilowatt-hour (c\$/kWh) during the period [11 pm - $7 \mathrm{am}), 9.7385 \mathrm{c} \$ /$ $\mathrm{kWh}$ during the periods [7 am $-10 \mathrm{am}),[3 \mathrm{pm}-6 \mathrm{pm}$ ) and $[9 \mathrm{pm}-11 \mathrm{pm})$, and $13.852 \mathrm{c} \$ / \mathrm{kWh}$ during the
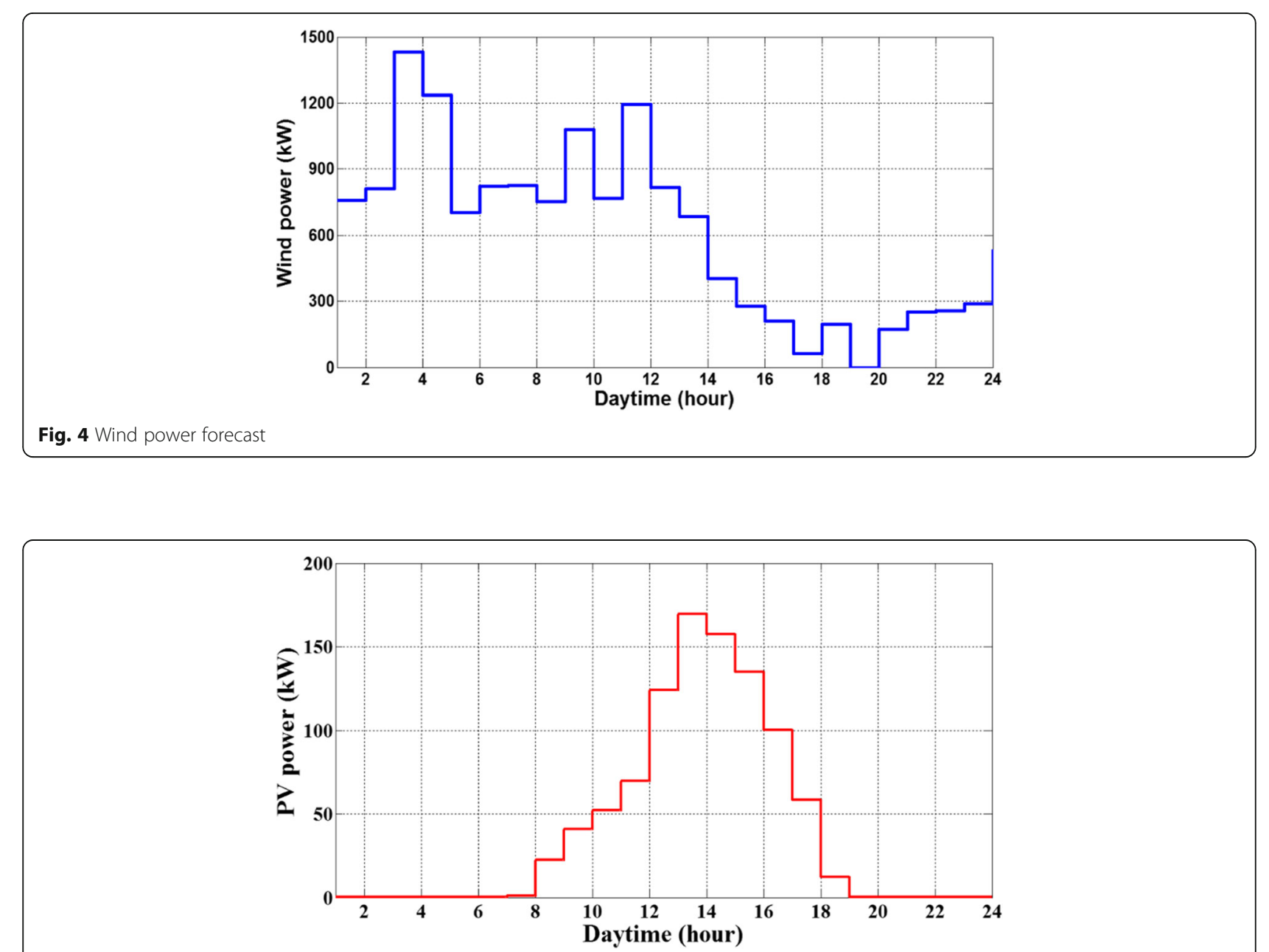

Fig. 5 PV solar power forecast 


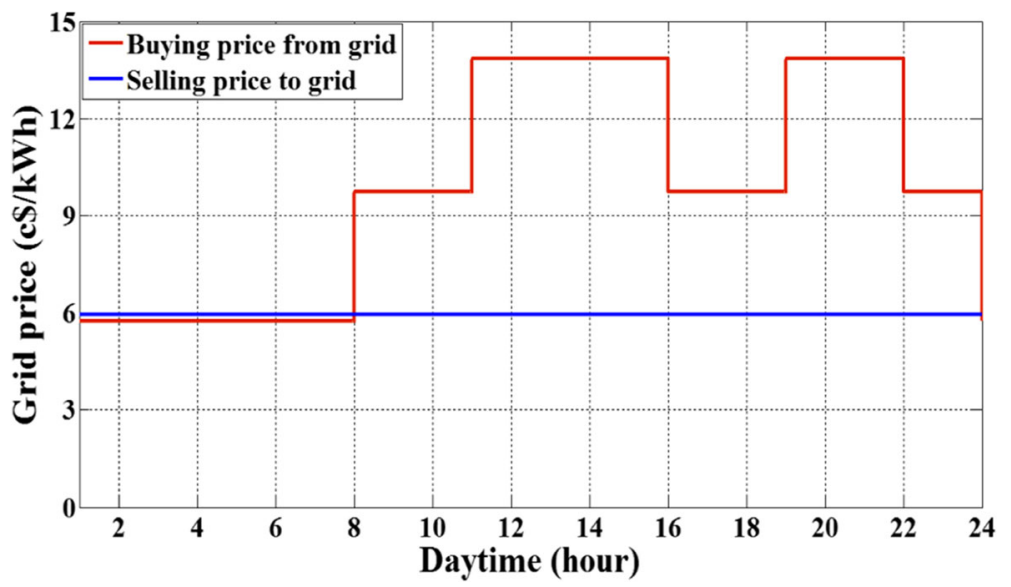

Fig. 6 Grid price for industrial companies in BDA, Beijing, in 2016

periods $[10 \mathrm{am}-3 \mathrm{pm})$ and $[6 \mathrm{pm}-9 \mathrm{pm})$. However, the price of selling power to the main grid is fixed throughout the day as $5.9492 \mathrm{c} \$ / \mathrm{kWh}$, shown in Fig. 6.

The operation and maintenance costs considered within the microgrid are, respectively $0.3767 \mathrm{c} \$ / \mathrm{kWh}$, $0.2169 \mathrm{c} \$ / \mathrm{kWh}, \quad 0.5767 \mathrm{c} \$ / \mathrm{kWh}, 0.003 \mathrm{c} \$ / \mathrm{kWh}$ and $0.0015 \mathrm{c} \$ / \mathrm{kWh}$ for the wind turbine system, PV systems, diesel generator, VRB and Li-Ion battery.

\subsection{Operation in isolated mode (case I)}

The day-ahead load demand forecast for this case is shown in Fig. 7. The peak load demand is less than the summation of the peak generation capacity of each DG unit.

The RegPSO-based optimal energy scheduling of the microgrid for the next day under isolated operation mode, and the corresponding SOCs of the ESUs are shown in Figs. 8 and 9, respectively.

As shown in the figures above, during the first $4 \mathrm{~h}$ [12 am - 4am) of the simulation period, there is a significant generation of wind energy and no generation from the PV source. In this period, the renewable energy completely supplies the load demands and charges the ESUs which were at minimum SOCs $(20 \%)$ before the simulation started, and the DE is off (zero power) to reduce the fuel cost as there is enough renewable generation in the microgrid. The ESUs continuously charge and their SOCs increases until 4 am, shown in Fig. 9. However, although they don't get fully charged the ESUs stop charging and their charging powers come to zero (inactive state) at 4 am since the available renewable generation can only supply the load demand since from this time till $1 \mathrm{pm}$.

During the period $[1 \mathrm{pm}-9 \mathrm{pm})$, the power generation from both the wind and PV sources is not enough to supply the load demands, and thus the ESUs start discharging to send power to the microgrid together with the wind, PV and DE.

The ESUs continuously discharge and reach their minimum storage capacity $(240 \mathrm{kWh}$ for VRB and $160 \mathrm{kWh}$ for Li-Ion battery), shown in Fig. 9, at 9 pm and their discharging power come zero then after. To reduce the cost of energy production, the ESUs are inactive state since then; until they will be charged again by an available

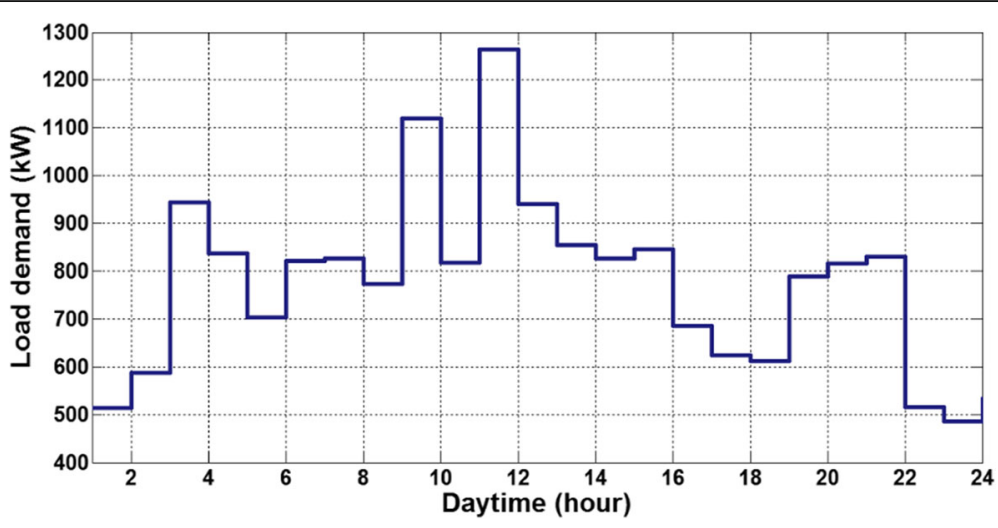

Fig. 7 Load demand forecast 


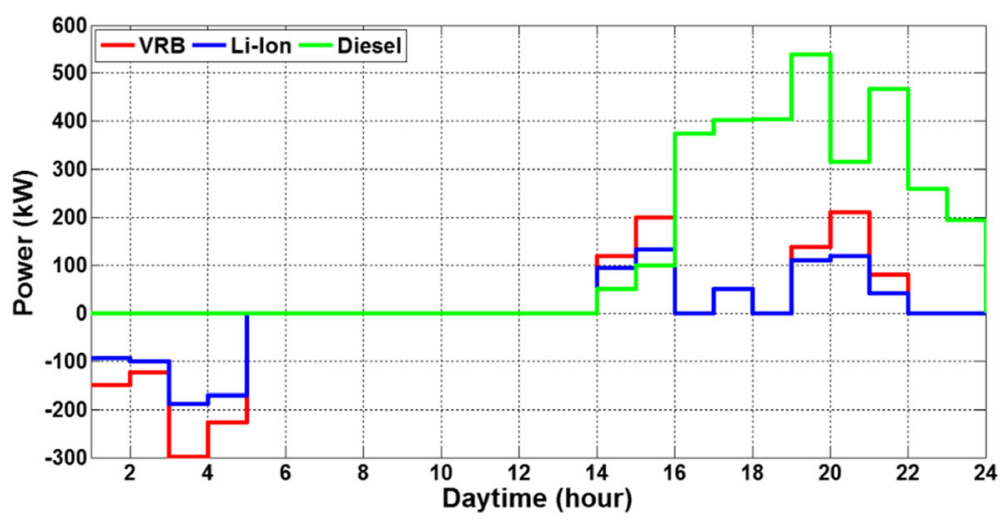

Fig. 8 Optimal dynamic scheduling using RegPSO in isolated mode

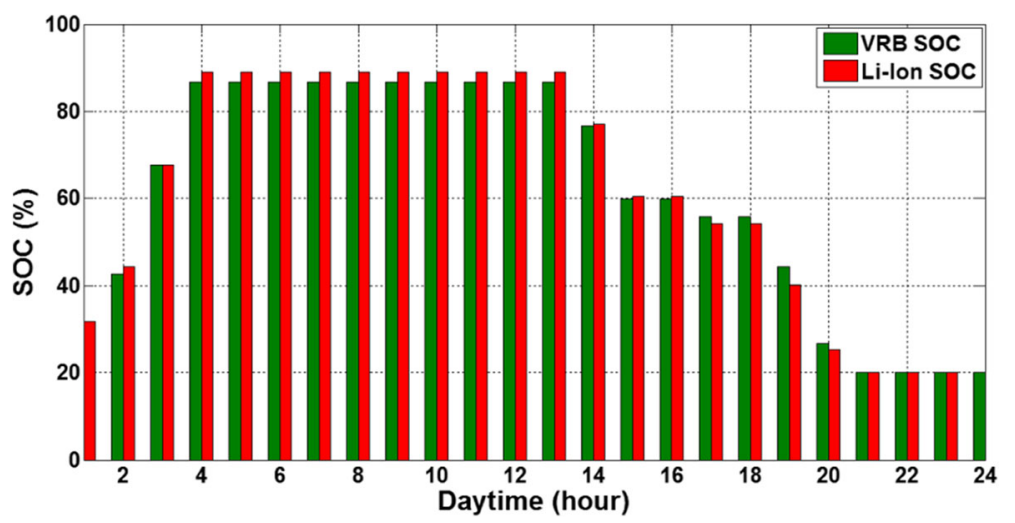

Fig. 9 SOCs of VRB and Li-lon battery obtained using RegPSO in isolated mode

excess renewable generations in the microgrid and their SOCs are kept at minimum value of $20 \%$. The wind and DE supply the load demands from $9 \mathrm{pm}$ to $12 \mathrm{am}$.

\subsection{Operation in Non-isolated mode (case II \& III)}

In this case, the microgrid is in a grid-connected mode, and participates in the energy trading exchange with the main grid in addition to supplying the load demands within it. Figure 10 shows the next day load demand forecast for this scenario.

Figure 11 shows the RegPSO-based optimal energy scheduling of the microgrid under grid-connected operation mode, and the corresponding SOCs of the ESUs are also shown in Fig. 12.

During the period [12 am $-6 \mathrm{am}$ ), shown in Fig. 11, the renewable energy completely supplies the load demands within the industrial park and charges the ESUs which were considered to be at their minimum SOC $(20 \%)$ before the simulation started at zero time (12 am). Moreover in this period, the microgrid sells the surplus generation to the main grid.
The ESUs continuously charge and reach their maximum storage capacity, shown in Fig. 12, at 6 am and then their charging power become zero. During the period [ $6 \mathrm{am}-1 \mathrm{pm})$, there is still an excess generation in the microgrid, however the ESUs are already fully charged, thus the microgrid keeps selling the excess energy to the main grid.

During cheap grid-price time period, the diesel fuel cost is more expensive than the grid price when the power is greater than $151 \mathrm{~kW}$. In moderate gridprice time slots, the diesel fuel cost is more expensive than the grid price when the power is greater than $311 \mathrm{~kW}$, and at the peak grid-price period, the diesel fuel cost is more expensive than the grid price when the power is greater than $472 \mathrm{~kW}$ as shown in Fig. 13.

The power generation from the renewables is not enough to supply the load demands and the grid price is peak (expensive) during the period $[1 \mathrm{pm}-3 \mathrm{pm}$ ). Hence, the ESUs start discharging to support the microgrid load demands together with the wind and PV and the $\mathrm{DE}$ and grid powers are zero in this period to minimize the total cost as shown Fig. 11. 


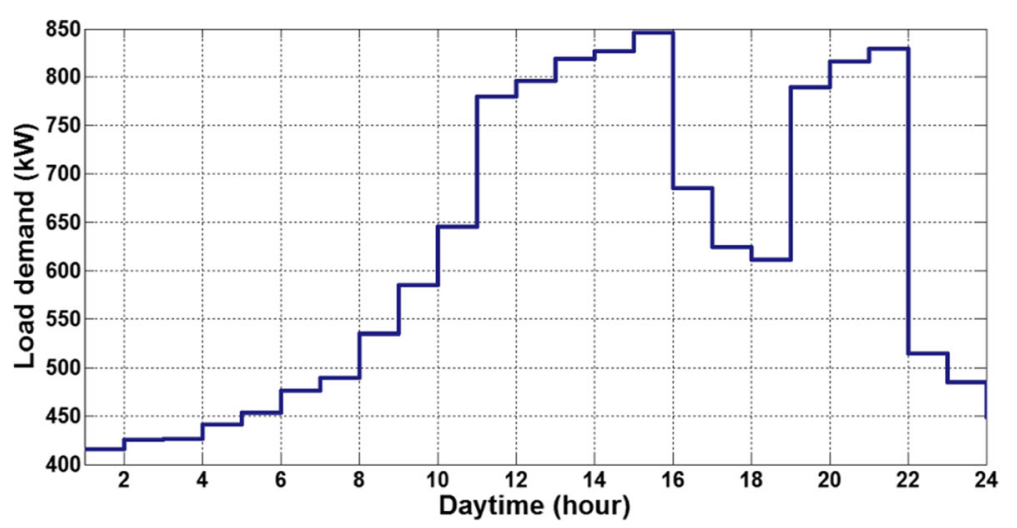

Fig. 10 Load demand forecast

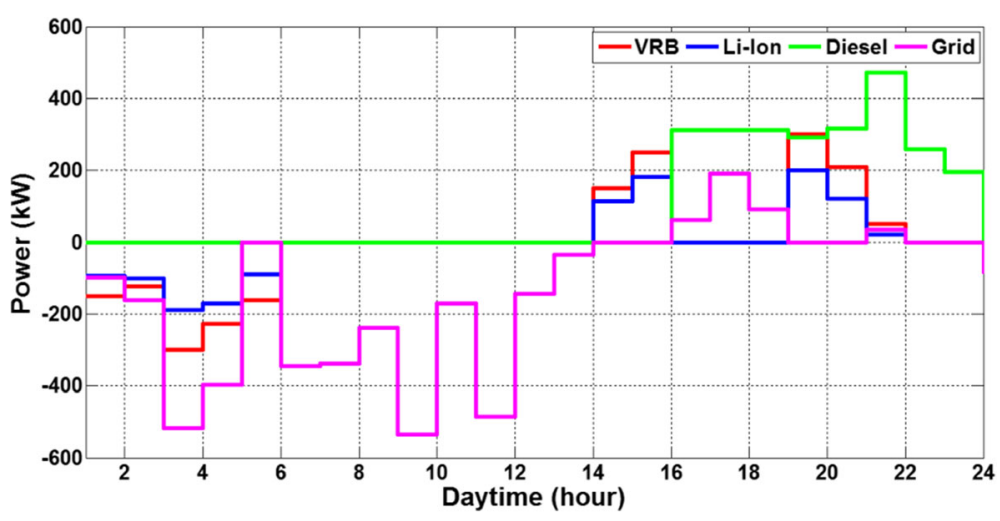

Fig. 11 Optimal dynamic scheduling using RegPSO in non-isolated operation mode

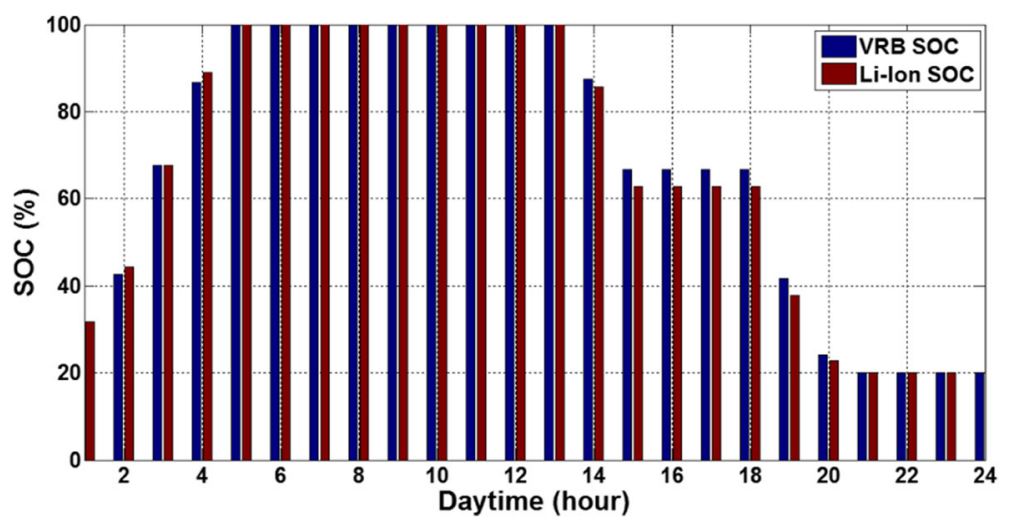

Fig. 12 SOCs of VRB and Li-lon battery obtained using RegPSO in non-isolated operation mode

During the period $[3 \mathrm{pm}-6 \mathrm{pm})$, the generation from the renewables is insufficient to supply the microgrid load demands and the grid price is moderate. Hence, the ESUs stop discharging for later peak hour demand use and the microgrid utilizes the generations from the DE and main grid for economic reasons as shown Fig. 11.
In the period $[6 \mathrm{pm}-9 \mathrm{pm})$, the microgrid load demand is greater than the local generations from the renewables. Since the electricity buying price is expensive in this period, the ESUs restart discharging to supply the load together with the wind and DE. The ESUs continuously discharge and reach their minimum storage 


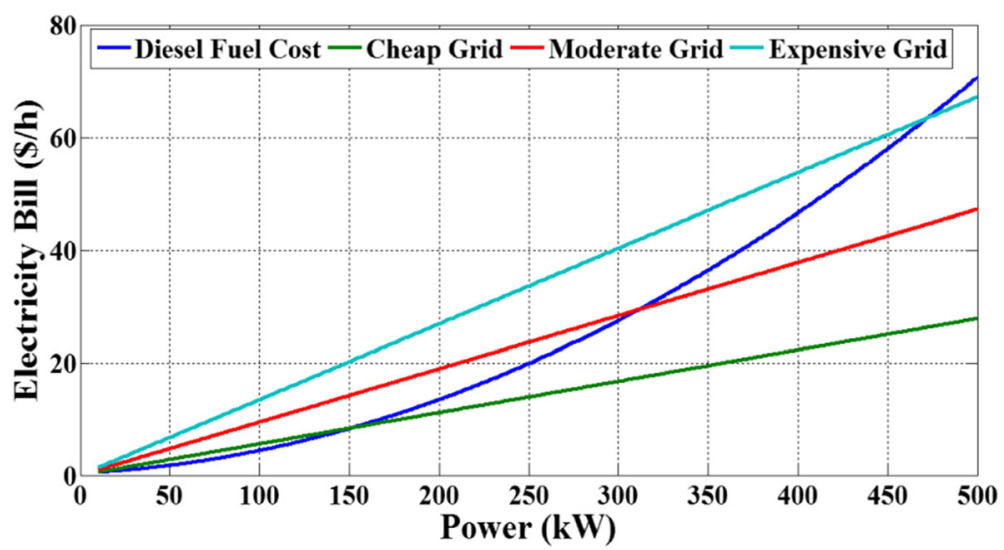

Fig. 13 Comparison of diesel fuel cost vs. time-varying grid-price

capacity $(240 \mathrm{kWh}$ for VRB and $160 \mathrm{kWh}$ for Li-Ion battery), shown in Fig. 12, at $9 \mathrm{pm}$ and their discharging power come zero then after. Thus, the ESUs are in inactive state since then; until they will be charged again by an available excess renewable generations in the microgrid and their SOCs are kept at minimum value of 20\% as shown in Fig. 12.
The load demand is supplied by the wind and DE during the period $[9 \mathrm{pm}-11 \mathrm{pm})$ and the grid power is zero for minimum cost. In the period [11 pm $-12 \mathrm{am}$ ), there comes again excess renewable generation from the wind source and the microgrid sells this energy to the main grid instead of starting charging the ESU for maximum daily total profit.

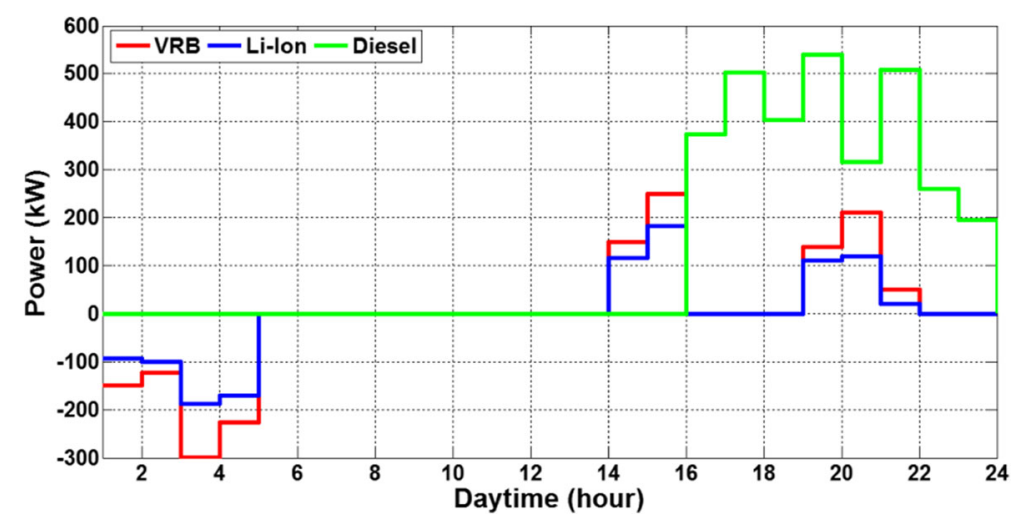

Fig. 14 Energy resources dynamic scheduling using GA in isolated operation mode

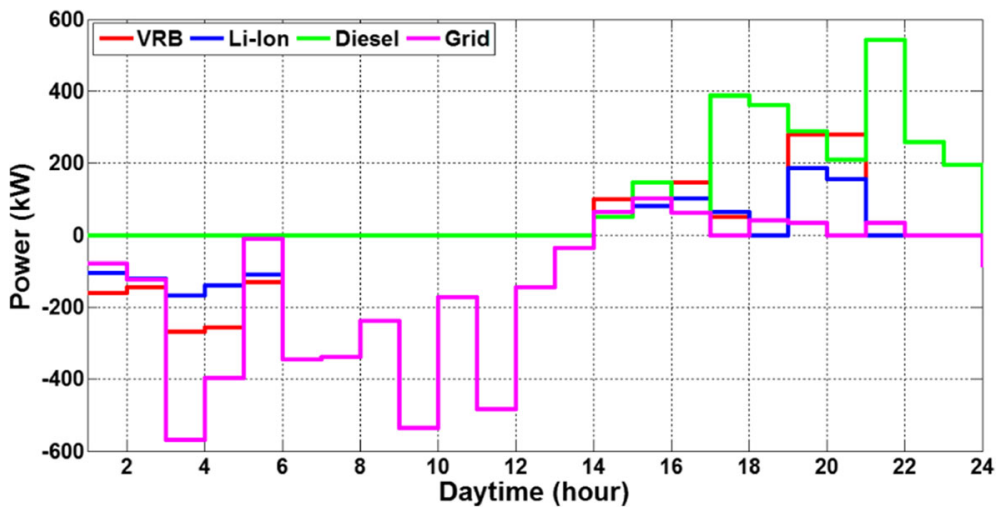

Fig. 15 Energy resources dynamic scheduling using GA in non-isolated operation mode 
Figures 14 and 15 show the GA-based optimal energy scheduling simulation results for the isolated and gridconnected operation modes, respectively.

The hourly values of energy production fuel costs by both optimization methods for the isolated operation mode is shown in Fig. 16. The hourly values of energy production fuel costs and grid power purchasing expenses obtained from both approaches for the gridconnected operation mode is shown in Fig. 17. The hourly values of energy selling revenues obtained from both approaches for the grid-connected operation mode is shown in Fig. 18.

As shown in Figs. 16 and 17, the microgrid hourly energy production and purchasing expenses are zero during the

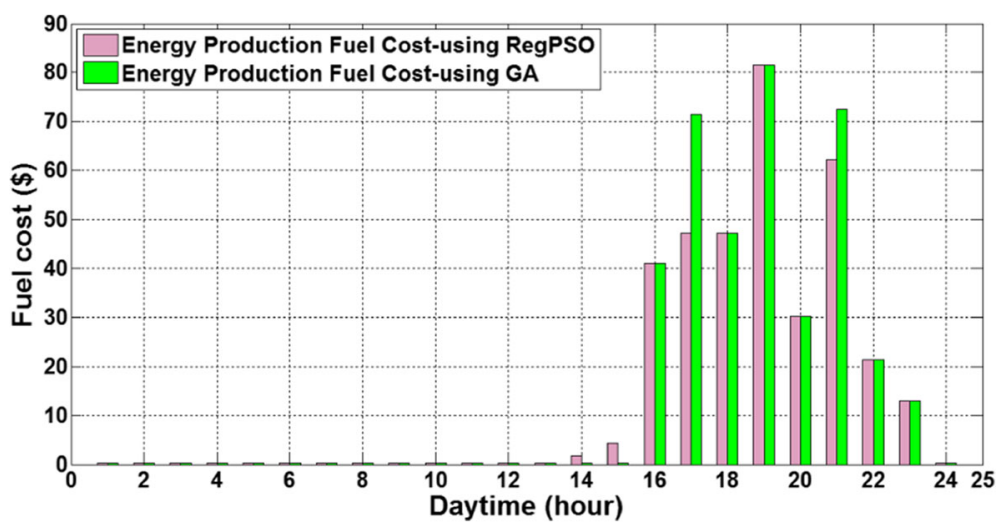

Fig. 16 Hourly comparison of energy production fuel costs in isolated mode of operation

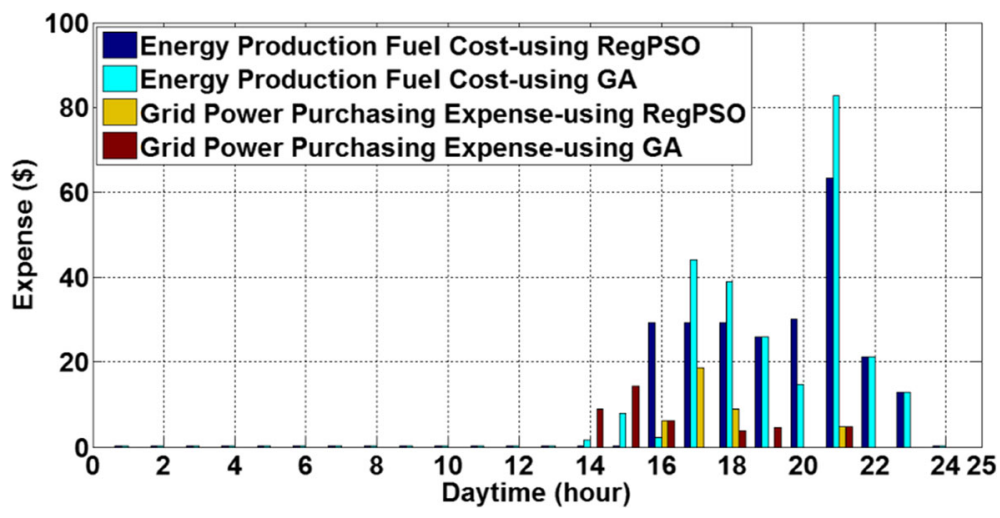

Fig. 17 Hourly comparison of energy production fuel costs and grid power purchasing expenses in non-isolated mode of operation

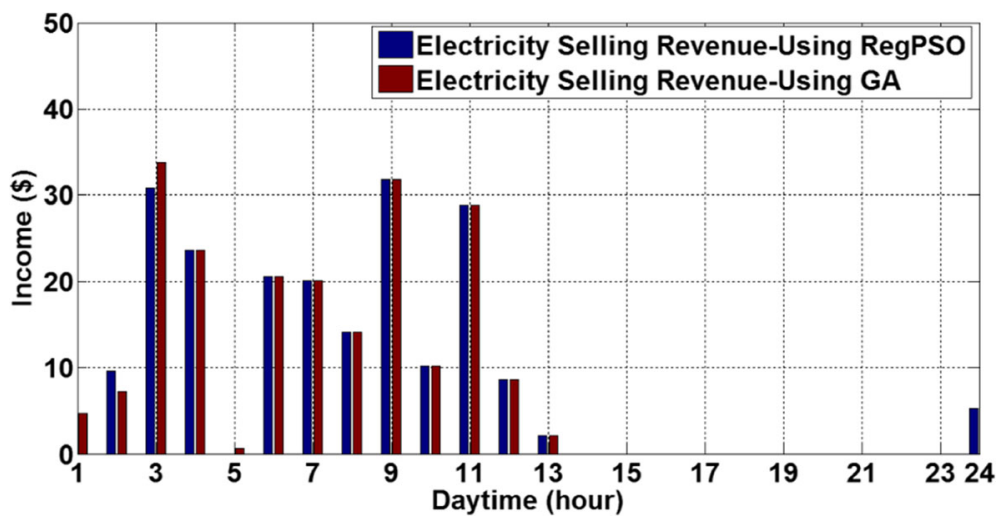

Fig. 18 Hourly comparison of energy selling revenue in non-isolated mode of operation 
Table 1 Fuel and energy trading costs by RegPSO and GA

\begin{tabular}{|c|c|c|c|c|c|c|}
\hline \multirow{3}{*}{$\begin{array}{l}\text { Optim. } \\
\text { Algorithm }\end{array}$} & \multicolumn{6}{|c|}{ Daily Total Cost (\$) } \\
\hline & \multicolumn{2}{|c|}{ Daily Energy Production Fuel Cost } & \multicolumn{2}{|c|}{ Daily Grid Power Purchasing Expense } & \multicolumn{2}{|c|}{ Daily Energy Selling Profit } \\
\hline & Isolated Mode & Non-isolated Mode & Isolated Mode & Non-isolated Mode & Isolated Mode & Non-isolated Mode \\
\hline RegPSO & 354.42 & 247.04 & 0 & 38.654 & 0 & 211.66 \\
\hline GA & 383.53 & 257 & 0 & 42.862 & 0 & 211.66 \\
\hline
\end{tabular}

Table 2 Computation time for RegPSO and GA

\begin{tabular}{lcr}
\hline Optimization & \multicolumn{2}{c}{ Total Computation Time (seconds) } \\
\cline { 2 - 3 } Algorithm & Isolated Mode & Non-isolated Mode \\
\hline RegPSO & 1.8678 & 2.1152 \\
GA & 14.9845 & 16.3456 \\
\hline
\end{tabular}

period [12 am - $3 \mathrm{pm}$ ) since the microgrid has sufficient renewable generation within it. The best performance of the RegPSO algorithm over the GA is clearly visible in most of the operation hours. Using RegPSO-based approach, the energy purchasing expense was very much lower than the GA-based purchasing prices. This shows the effectiveness of the proposed strategy for achieving a global optimum solution as desired. Moreover, as it is shown in Table 1, the RegPSO-based energy storage management strategy has given a lower daily total expense of energy production and purchasing than the GA-based approach.

As seen in Fig. 18, during the period [12 am - $1 \mathrm{pm}$ ), the microgrid sells energy to the main grid and gets profit. After $1 \mathrm{pm}$, except at [11 pm - $12 \mathrm{am})$, the microgrid has no surplus generation to sell, and hence the selling income is zero. Moreover, since the electricity selling price to the main grid is fixed throughout the day, the hourly selling income values obtained by both algorithms (RegPSO and GA) are almost the same.

Table 2 gives the total computation time taken by both energy management optimization approaches (using Intel core i5-5200 CPU, $2.20 \mathrm{GHz}$ processor and $4 \mathrm{~GB}$ RAM PC) for both microgrid operation modes. The RegPSO-based energy storage management has allocated the schedule within a short period of time compared to the GA-based method in both operation modes.

\section{Conclusion}

Optimal dynamic energy scheduling strategy for a Wind-PV-DE-VRB-Li-Ion industrial microgrid under both isolated and grid-tied operation modes was proposed in this study using the RegPSO algorithm. The proposed approach takes into account the fluctuations of renewables and load demands in the microgrid and appropriate day-ahead forecasts have been made to overcome these fluctuations. Simulation results have demonstrated the effectiveness and possible advantages of the developed energy management strategy in minimizing the energy production fuel cost, grid power purchasing expense, maximizing the energy selling profit, maximizing the economic usage of ESUs and enhancing the utilization of the renewables within the microgrid. Comparison of simulation results with GA-based approach, demonstrated the effectiveness of the proposed RegPSObased energy management strategy in resulting a possible reduced energy production fuel cost and grid power purchasing expense for the microgrid. Moreover, the proposed approach is fast convergent and results global optimum solutions in an acceptable short computation time. This also manifests the ability of the proposed approach for real time energy management of microgrids with any number of renewable DGs and ESUs under both operation modes.

\section{Authors' contributions \\ Han Li contributed to the conception of the study. Abinet Tesfaye Eseye contributed significantly to analysis, manuscript preparation and manuscript submission as a corresponding author; Jianhua Zhang and Dehua Zheng helped perform the study analysis with constructive discussions, senior professional advice and revised the manuscript. All authors read and approved the final manuscript.}

\section{Competing interests}

The authors declare that they have no competing interests.

\section{Author details}

${ }^{1}$ Power Grid Energy Saving and Building Energy Conservation Department, State Grid Energy Conservation Service Co., Ltd., Beijing 100052, CN, China. ${ }^{2}$ School of Electrical and Electronic Engineering, North China Electric Power University, Changping District, Beijing 102206, CN, China. ${ }^{3}$ Goldwind Science and Etechwin Electric Co., Ltd., Beijing Economic-Technology Development Area, Beijing 100176, CN, China.

Received: 7 December 2016 Accepted: 28 February 2017

Published online: 08 April 2017

\section{References}

1. Velik, R., \& Nicolay, P. (2014). Grid-price-dependent energy management in microgrids using a modified simulated annealing triple-optimizer. App/ Energy, 130, 384-395.

2. Velik, R. (2013). "The influence of battery storage size on photovoltaics energy self-consumption for grid-connected residential buildings." IJARER International Journal of Advanced Renewable Energy Research, 2(6). 
3. Velik, R. (2013). "Battery storage versus neighbourhood energy exchange to maximize local photovoltaics energy consumption in gridconnected residential neighbourhoods." IJARER International Journal of Advanced Renewable Energy Research, 2(6).

4. Velik, R (2013). "Renewable energy self-consumption versus financial gain maximization strategies in grid-connected residential buildings in a variable grid price scenario." IJARER International Journal of Advanced Renewable Energy Research, 2(6).

5. Chen, Y., Lu, S., Chang, Y., Lee, T., \& Hu, M. (2013). Economic analysis and optimal energy management models for microgrid systems: a case study in Taiwan. Appl Energy, 103, 145-154.

6. Gregoratti, D., \& Matamoros, J. (2013). Distributed convex optimization of energy flows: the two-microgrid case. In 1st international black Sea conference on communication and networking (pp. 201-205).

7. Celli, G., Pilo, F. Pisano, G., \& Soma, G. (2005). Optimal participation of a microgrid to the energy market with an intelligent EMS. In 7th international power engineering conference (pp. 663-668).

8. Livengood, D., \& Larson, R. (2009). The energy Box: locally automated optimal control of residential electricity usage. Serv Sci, 1(1), 1-16.

9. Mashhour, E., \& Moghaddas-Tafreshi, S. (2010). Integration of distributed energy resources into low voltage grid: A market-based multiperiod optimization model. Electr Pow Syst Res, 80(4), 473-480.

10. Kriett, P., \& Salani, M. (2012). Optimal control of a residential microgrid. Energy, 42(1), 321-330

11. Malysz, P., Sirouspour, S. and Emadi, A. (2014). "An Optimal Energy Storage Control Strategy for Grid-connected Microgrids." IEEE Transactions on Smart Grid, 5(4). July.

12. Zhao, B., Zhang, X., Li, P., Wang, K., Xue, M., \& Wang, C. (2014). Optimal sizing, operating strategy and operational experience of a standalone microgrid on Dongfushan Island. Appl Energy, 113, 1656-1666.

13. Pourmousavi, S. A., Nehrir, M. H., Colson, C. M., and Wang, C. (2010). "Realtime Energy management of a Stand-Alone Hybrid Wind-Microturbine Energy System Using Paricle Swarm Optimization." IEEE Transactions on Sustainable Energy, 1(3). October.

14. Aric James, L. (2013). Real-time energy management of an islanded microgrid using multi-objective particle swarm optimization. Bozeman: Master's Thesis, Electrical Engineering, Montana State University.

15. Evers, G. I., \& Ghalia, M. B. (2009). Regrouping particle swarm optimization: a New global optimization algorithm with improved performance consistency across benchmarks. In IEEE international conference on systems, Man and cybernetics (SMC) (pp. 3901-3908).

16. Zheng, Z. (2012). Optimal energy management for microgrids. South Calorina: PhD Dissertation Electrical and Computer Engineering, Clemson University, Clemson.

17. Borghetti, A., Bosetti, M.,and Grillo, S. (2010). "Short-term scheduling and control of active distribution systems with high penetration of renewable resources." IEEE Systems Journal, 4(3). September

18. Del Valle, Y., Venayagamoorthy, G. K., Mohagheghi, S., Hernandez, J.-C.. \& Harley, R. G. (2008). Particle swarm optimization: basic concepts, variants and applications in power systems. IEEE Trans Evol Comput, 12(2), 171-195.

19. Evers, G. (2016). PSO Research Toolbox Documentation (Version: 2016-0308), M.S. thesis code documentation. http://www.georgeevers.org/pso_ research_toolbox_documentation.pdf. Accessed 8 Mar 2016.

\section{Submit your manuscript to a SpringerOpen ${ }^{\circ}$ journal and benefit from:}

- Convenient online submission

$\checkmark$ Rigorous peer review

- Immediate publication on acceptance

- Open access: articles freely available online

- High visibility within the field

- Retaining the copyright to your article 